\title{
OPEN Diverse classes of constraints enable broader applicability of a linear programming-based dynamic metabolic modeling framework
}

\author{
Justin Y. Lee \& Mark P. Styczynski ${ }^{\circledR}$
}

Current metabolic modeling tools suffer from a variety of limitations, from scalability to simplifying assumptions, that preclude their use in many applications. We recently created a modeling framework, Linear Kinetics-Dynamic Flux Balance Analysis (LK-DFBA), that addresses a key gap: capturing metabolite dynamics and regulation while retaining a potentially scalable linear programming structure. Key to this framework's success are the linear kinetics and regulatory constraints imposed on the system. However, while the linearity of these constraints reduces computational complexity, it may not accurately capture the behavior of many biochemical systems. Here, we developed three new classes of LK-DFBA constraints to better model interactions between metabolites and the reactions they regulate. We tested these new approaches on several synthetic and biological systems, and also performed the first-ever comparison of LK-DFBA predictions to experimental data. We found that no single constraint approach was optimal across all systems examined, and systems with the same topological structure but different parameters were often best modeled by different types of constraints. However, we did find that when genetic perturbations were implemented in the systems, the optimal constraint approach typically remained the same as for the wild-type regardless of the model topology or parameterization, indicating that just a single wild-type dataset could allow identification of the ideal constraint to enable model predictivity for a given system. These results suggest that the availability of multiple constraint approaches will allow LK-DFBA to model a wider range of metabolic systems.

Mathematical and computational models are often used to study metabolism, the set of reactions that supply the chemical precursors necessary for almost all cellular processes. These metabolic models are significantly cheaper and faster to run than laboratory experiments, meaning that they can be of tremendous value when they are able to predict how changes in or to a metabolic system can affect its state. While a few pathways and sections of metabolism (e.g., glycolysis and central carbon metabolism) have been modeled and characterized quite well in a few organisms (e.g., Saccharomyces cerevisiae and Escherichia coli) ${ }^{1,2}$, genome-scale models that capture the kinetics of metabolism at a systems scale have been more difficult to develop. Metabolism involves many interconnected reactions and pathways, making it critical to include as much of metabolism as possible in metabolic models to better represent the system and generate accurate predictions. Metabolomics, which is the systems-scale measurement of metabolites in biological systems, thus has great potential to provide the information necessary to drive systems-scale metabolic models since metabolomics data are easier to acquire than, for example, metabolic flux data. However, creating genome-scale metabolic models that capture critical system behaviors like metabolic dynamics remains an outstanding challenge in the field, which has prevented the value of metabolomics data in this context from being fully realized.

The most popular type of frameworks for metabolic modeling are constraint-based models-including flux balance analysis (FBA) 3,4 and ordinary differential equation (ODE) models. FBA assumes that the metabolic system is at steady state, which allows it to be modeled as a linear program (LP) that can be efficiently solved 
(even at the genome scale) but precludes modeling metabolite dynamics without substantial changes to the framework. ODE models are more widely used when dynamics are important, but are typically limited to smallerscale modeling of well-studied pathways (e.g. central carbon metabolism ${ }^{1}$ or glycolysis $^{5}$ ) and the best-studied organisms (e.g. $\mathrm{CHO}$ cells ${ }^{6}$ ) due to uncertainty in the mathematical form and parameter values for the reaction kinetics terms. Only a few exceptions ${ }^{7-10}$ have approached genome-scale ODE models, and they still require lengthy parameter estimation steps, prior information about kinetic constants, or have only been shown to be useful near the reference state of the system. As a result, steady-state fluxes continue to be the almost exclusive focus of study for genome-scale models. Modeling frameworks that can predict various metabolic phenotypes at the genome scale in a computationally tractable way have great potential for understanding, predicting, and controlling metabolism.

To address this problem, we recently developed Linear Kinetics-Dynamic Flux Balance Analysis (LK-DFBA), a modeling strategy to efficiently track metabolite dynamics ${ }^{11}$. LK-DFBA combines advantages of both constraintbased and ODE models, unrolling the temporal aspect of the system into a larger stoichiometric matrix that captures metabolite dynamics while retaining a LP structure. The most critical element to accomplishing this goal is the addition of linear kinetics constraints that model the interactions between metabolites and the reactions whose fluxes they affect, including mass action kinetics and allosteric regulatory interactions. The use of these constraints requires the inclusion of some kinetic parameters, a disadvantage that results from the effort to capture kinetics (as an ODE model does) rather than steady state; however, the number of parameters in LK-DFBA that need to be estimated can be fewer or easier to estimate than in ODE models due to these linear kinetics constraints, which can partially ameliorate that disadvantage. This point is relevant since the difficulty in estimating a large number of kinetic parameters is one of the key reasons why it is a significant challenge to develop ODE-based models of large systems. Furthermore, other modeling frameworks that retain linear structures, as LK-DFBA does, can be used with many existing metabolic modeling tools that require constraint-based models, such as OptKnock ${ }^{12}$; the application of LK-DFBA with such modeling tools is a potentially promising future direction. We have previously shown that LK-DFBA can outperform ODE-based modeling approaches when used in conditions most relevant to metabolomics data (low sampling frequency and high noise) ${ }^{11}$. A framework such as LK-DFBA that can be shown to model systems at the genome scale is essential to take full advantage of metabolomics data.

In our initial description of LK-DFBA, we explored two different approaches for model parameterization. The first approach, LK-DFBA (LR), parameterizes constraints solely via linear regression of interacting metabolite concentration and flux data. The second approach, LK-DFBA $(\mathrm{LR}+)$, uses the parameters from the linear regressions as initial seeding values for a secondary optimization to identify the optimal constraints for each interaction. While LK-DFBA (LR +) yields better fits to training data than LK-DFBA (LR), the latter approach estimates its parameters with trivial computational effort while still producing results that are similar in error to ODE models. As the systems being studied get larger, the LK-DFBA (LR +) approach may become computationally intractable for some systems at a certain size, and so LK-DFBA (LR) may be the preferable approach for the efficient construction and parameterization of metabolic models at the genome scale.

However, the overall LK-DFBA framework still has some limitations in terms of how accurately it represents the underlying biology and biochemistry of the system. For example, the linear kinetics constraints used in LKDFBA (LR) may be viewed as crude approximations of the interactions between metabolites and fluxes, which are typically non-linear in nature. While kinetic equations found in ODE models (such as Michaelis-Menten or biochemical system theory (BST) representations ${ }^{13,14}$ ) can capture the non-linearity of these interactions, the current linear framework in LK-DFBA cannot. Additionally, when allosteric regulatory information is considered (which LK-DFBA includes in its framework), reaction fluxes are often controlled by multiple metabolites. Currently, LK-DFBA creates separate constraints for each metabolite that controls a flux, which precludes modeling how multiple metabolites simultaneously interact with a reaction flux.

Since the linear kinetics constraints are so critical in LK-DFBA's functioning, it is likely that improving those constraints could have a substantial impact on LK-DFBA's ability to capture and predict biological phenomena. Accordingly, we devised three new types of kinetics constraints for LK-DFBA to account for biologically important features like non-linearity and simultaneous regulation by multiple metabolites. These new approaches were compared to the original LK-DFBA (LR) constraints by testing on synthetic model systems as well as models based on Lactococcus lactis and Escherichia coli ${ }^{1,2}$. We found that the ideal constraint for a given model depended not only on its structure, but also on its set of parameter values even for the same structure. The new types of constraints allowed improved fitting of data in many tested models. We then probed these constraint approaches for their robustness to model perturbation and their ability to predict phenomena not captured in training data. We found that while different model topologies and parameter sets have different optimal constraint approaches, the same constraint approach was optimal across the majority of perturbations for any given model (overall, 70\% across all models and noise conditions reported here), indicating that just one set of data is sufficient for parameterization robust enough for predictions of model perturbations. We also showed that the LK-DFBA approach chosen for the L. lactis and E. coli models can be used to predict changes in several critical metabolites and fluxes in qualitative agreement with literature experimental results. The new constraint strategy and its robustness to model perturbations have promise for moving the LK-DFBA framework towards modeling increasingly large metabolic systems in the future.

\section{Methods}

LK-DFBA. Linear Kinetics-Dynamics Flux Balance Analysis (LK-DFBA) is a recently developed modeling strategy that is capable of capturing metabolite dynamics and potentially scalable. The full details of this approach have been described in detail previously ${ }^{11}$, so we only outline the most important aspects of our framework here. 
In brief, LK-DFBA is a linearly-constrained quadratic program with temporal dynamics modeled by discretizing time and unrolling the system into a larger matrix $A$ representing each time point separately. $A$ consists of the stoichiometric matrix of the system and an identity matrix, which when multiplied by $\vec{w}$ (a vector of fluxes $\vec{v}$ and pooling fluxes $\overrightarrow{v_{p}}$ (i.e. $\left.\frac{\mathrm{d} x}{\mathrm{dt}}\right)$ ), calculate the system's mass balances at each time point $t_{k}$. The fluxes are constrained by lower $\left(\vec{w}_{L B}\right)$ and upper $\left(\vec{w}_{U B}\right)$ bounds. LK-DFBA includes a quadratic objective function $z$ where $\lambda$ is a small penalty on the norm of the solution vector $\vec{\omega}$ and $\vec{c}$ is a vector of weights. While LK-DFBA is technically a quadratic program, we found that adding this penalty reduced the possibility of solution degeneracy and led to no appreciable increase in computation time compared to a linear objective function. The solution vector consists of fluxes $\vec{v}$ and metabolite concentrations $\vec{x}$ at each timepoint in the unrolled model. Like the fluxes, the metabolite concentrations are also constrained by lower $\left(\vec{x}_{L B}\right)$ and upper $\left(\vec{x}_{U B}\right)$ bounds. Linear inequality constraints that model mass action kinetics and metabolite-dependent regulation are included in the model; they are the driving force behind metabolite accumulation and depletion by limiting the maximum flux $v_{i}$ allowed based on the availability of metabolites $x_{j}$ over time. In the LK-DFBA (LR) approach, these linear kinetics constraints are modeled using linear regression on assumed available metabolomics and fluxomics data to estimate $a$ and $b$ parameters for each of the $n$ pairings of metabolites that participate in a flux reaction. If fluxomics data are unavailable, dynamic flux estimation (DFE) can be used to infer flux values from concentration data ${ }^{15}$. However, estimated fluxes that are far from their true values can significantly impact the accuracy of LK-DFBA and a substantial amount of metabolomics data is recommended before using the DFE approach. The formulation of an LK-DFBA model is presented in Eq. (1).

$$
\begin{aligned}
& \max _{\vec{\omega}} z=\vec{c}^{T} \vec{\omega}-\lambda \vec{\omega}^{T} \vec{\omega} \\
& \text { s.t. } 0=A \vec{w}\left(t_{k}\right) \\
& \vec{w}_{L B} \leq \vec{w}\left(t_{k}\right) \leq \vec{w}_{U B} \\
& \vec{x}_{L B} \leq \vec{x}\left(t_{k}\right) \leq \vec{x}_{U B}^{U B} \\
& \vec{x}\left(\mathrm{t}_{0}\right)=\vec{x}_{0} \\
& x_{i}\left(t_{k}\right)=x_{i}\left(t_{k-1}\right)+\Delta t \cdot v_{p, i}\left(t_{k}\right) \\
& \sum_{i} v_{i, n}\left(t_{k+1}\right) \leq b_{n}+a_{n} \sum_{j} x_{j, n}\left(t_{k}\right) \\
& \forall k \in(1, n T), \forall i \in\{v\}_{n}, \forall j \in\{x\}_{n}, \forall n \in\left(1, n_{r}\right)
\end{aligned}
$$

where

$$
\begin{aligned}
A \vec{w} & =[S-I]\left[\begin{array}{c}
\vec{v} \\
\vec{v}_{p}
\end{array}\right] \\
\vec{\omega} & =\left[\vec{w}^{T}\left(t_{1}\right), \ldots, \vec{w}^{T}\left(t_{n T}\right), \vec{x}^{T}\left(t_{0}\right), \vec{x}^{T}\left(t_{1}\right), \ldots, \vec{x}^{T}\left(t_{n T}\right)\right]^{T}
\end{aligned}
$$

In the LK-DFBA $(\mathrm{LR}+)$ method, the parameters from the LK-DFBA (LR) approach are used as initial estimates in a secondary optimization step that finds improved kinetics constraint parameters, though at the cost of computational time. Because LK-DFBA retains a simple QP structure with entirely linear constraints, it is potentially scalable and could be further developed to be used with current constraint-based modeling tools. The objective functions used for each individual model are described in the Supplementary Methods.

Constraint approaches. $L K-D F B A(L R)$. The original LK-DFBA approach uses linear kinetics constraints to model the interaction between a metabolite and a flux, parameterized using linear regression on available metabolomics and fluxomics data. These constraints take the form of $v \leq a x+b$, where $v$ is the flux being constrained, $x$ is the concentration of a metabolite that interacts with the flux, and $a$ and $b$ are the linear constraint parameters. These interactions may be due to mass action kinetics, where the interactions are known based on the stoichiometric topology of the system, or they may stem from allosteric regulation. While we have previously shown that these linear approximations of metabolic interactions can be effective for modeling metabolism, they are still approximations of the true non-linear and interconnected biochemical relationships in metabolism. Below, we discuss three new constraint approaches to address these potential limitations.

LK-DFBA (NLR). While the key advantage of using constraint-based models is their LP structure that enables efficient identification of the optimal solution of the problem, most metabolite-flux interactions exhibit non-linear behavior that may not be captured well by linear equations. Recently, computational solvers have improved such that quadratically constrained programs (QCPs) are not much more computationally expensive than LPs. Accordingly, we implemented quadratic constraints into the LK-DFBA framework to explore their potential for improving model accuracy with only a modest increase in computational time. One important aspect of LPs and QCPs is that all of the constraints must create a convex feasible solution space to guarantee that a global optimum can be found ${ }^{16}$. If $v \leq a x^{2}+b x+c$ represents a quadratic constraint, where $v$ is the flux being constrained, $x$ is the concentration of a metabolite that interacts with $v$, and $a, b$, and $c$ are the parameters of the quadratic constraint, $a$ must be a negative value to retain a convex solution space. If $a$ is found to be a positive value during parameterization, we convert the quadratic constraint into its original linear form as found 


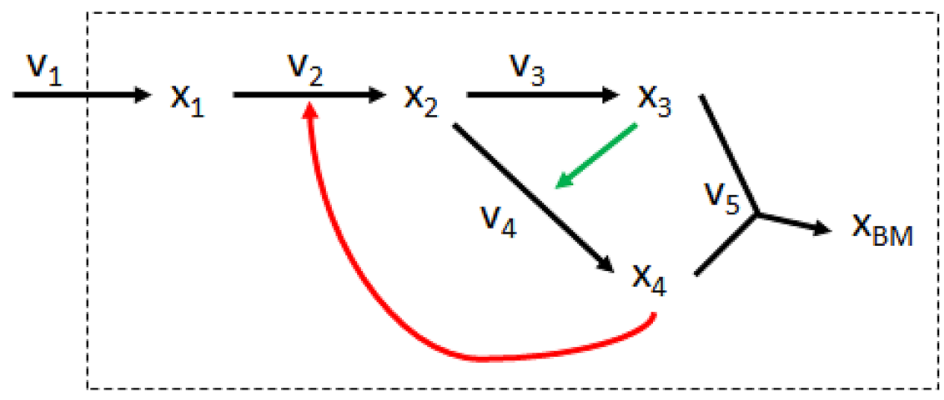

$$
\begin{aligned}
& \text { ODE equations: } \\
& v_{1}=1 \\
& v_{2}=a_{2} x_{1}{ }^{b 21} x_{4}{ }^{b 2 r 4} \\
& v_{3}=a_{3} x_{2}{ }^{632} \\
& v_{4}=a_{4} x_{2}{ }^{b 42} x_{3}{ }^{b 4 r 3} \\
& v_{5}=a_{5} x_{3}{ }^{b 53} x_{4}{ }^{b 54}
\end{aligned}
$$

Figure 1. Synthetic model. Adapted from another branched pathway model used in previous work ${ }^{11} \cdot \mathrm{v}_{1}, \mathrm{v}_{2}, \mathrm{v}_{3}$, $\mathrm{v}_{4}$, and $\mathrm{v}_{5}$ are system fluxes (black arrows) and $\mathrm{x}_{1}, \mathrm{x}_{2}, \mathrm{x}_{3}, \mathrm{x}_{4}$, and $\mathrm{x}_{\mathrm{BM}}$ are metabolites, where $\mathrm{x}_{\mathrm{BM}}$ is a metabolite representing biomass. Green and red arrows represent positive and negative regulatory interactions, respectively. ODE equations for the model are shown in the inset, where blue $a$ and $b$ parameters are mass action kinetic parameters and green and red $b$ parameters are positive and negative regulatory parameters, respectively.

in LK-DFBA (LR). The parameters were estimated using linear least squares regression. We refer to this overall approach as LK-DFBA (NLR).

LK-DFBA (DR). Enzymatic reactions are often controlled by more than a single metabolite that can either induce or inhibit enzyme activity, which should ideally be captured in the model constraints. To model such regulation of a reaction by multiple metabolites, LK-DFBA (LR) creates individual linear constraints for each controller metabolite that are independent of each other and are thus unable to capture the synergistic or antagonistic effects of multiple metabolites working in conjunction to regulate a flux. We implemented a new strategy that uses dimensionality reduction to consolidate information content from all controller metabolites for a flux into a single constraint. Dimensionality reduction is often used in data analysis, including analysis of metabolomics data, to more easily represent and digest datasets with many measured variables. Principal component analysis (PCA) is one of the most commonly used dimensionality reduction approaches; it linearly transforms the original variables into new, orthogonal composite variables called principal components that capture as much variance in the original variable data in as few principal components as possible ${ }^{17}$. Ideally, the first or first few principal components capture the majority of the variance in the original dataset, which allows one to analyze only those composite variables rather than all of the original variables at once. Here, we use PCA to capture the maximal variance of the controller metabolite data in a single principal component and use that composite variable as the regressor during linear regression with the target flux data. These new constraints are represented as $v \leq a P C_{1}+b$, where $v$ is the flux being constrained, $P C_{1}$ is the controller metabolite concentration data projected into the first principal component, and $a$ and $b$ are the constraint parameters. The parameters were estimated using linear least squares regression. We refer to this dimensionality reduction approach as LK-DFBA (DR).

LK-DFBA (HP). Another approach we used to model interactions of a flux with multiple metabolites was hyperplane constraints. Unlike LK-DFBA (DR), which always builds constraints in two dimensions (i.e. the target flux vs. the first principal component), the hyperplane constraint exists in $(n+1)$ dimensions, where $n$ is the number of metabolites that control a target flux. This approach may avoid loss of information content from metabolite data as is possible during dimensionality reduction: as the number of metabolites in an interaction increases, the likelihood of the first principal component not capturing the majority of variance in the data increases. The hyperplane constraint equation can be represented as $v \leq b+\sum_{i=1}^{n} a_{i} x_{i}$, where $v$ is the flux being constrained, $n$ is the number of metabolites that interact with $v, x_{i}$ is the concentration of metabolite $i, a_{i}$ is the constraint parameter for metabolite $x_{i}$, and $b$ is another constraint parameter. The parameters were estimated using linear least squares regression. We refer to the hyperplane approach as LK-DFBA (HP).

Test models. Synthetic model. The first system we examined was a simple synthetic model with five metabolites and five fluxes that was derived from a branched pathway model used previously ${ }^{11}$. This system is represented via an ODE-based model that uses power-law kinetics to represent reaction fluxes ${ }^{14}$. The kinetic equations for each pathway are shown in Fig. 1. To create a variety of synthetic models with the same stoichiometric topology, we randomly generated $a$ and $b$ parameters in each kinetic equation. The parameters for each model can be found in Table S1. Time course metabolite and flux data were generated by solving the ODE system in MATLAB (2018b).

Lactococcus lactis model. This model was created by Costa et al. and comprises central metabolism and production pathways for important metabolites such as mannitol and 2,3-butanediol ${ }^{2}$. The L. lactis model consists of 26 metabolites and 21 fluxes and is publicly available on KiMoSys ${ }^{18}$. Noiseless data were generated in COPASI 4.24 (Build 197) using the default initial conditions and parameters over a simulation time of two hours. 
Escherichia coli model. The E. coli model developed by Chassagnole et al. encompasses glycolysis and the pentose phosphate pathway ${ }^{1}$. This model is publicly available on KiMoSys, but was rebuilt within MATLAB to allow easy creation of new models that use the original E. coli model's topology and stoichiometry. Noiseless data for the original E. coli model were generated in MATLAB (2018b) using the default initial conditions and parameters, while random initial conditions and parameters were used for the new models with the E. coli topology. To be consistent with our previous work, we used a simulation time of ten seconds ${ }^{11}$ to generate ODE data used to fit the constraint parameters in the LK-DFBA models. More information about model parameters, the objective functions used for each model, and additional implementation details can be found in the Supplementary Methods.

Pathway perturbations. To test the ability of LK-DFBA to predict metabolic behaviors not represented in the training data, we introduced perturbations into each system either through down-regulation (indicated with the prefix ' $\mathrm{d}$ ' in all figures) or up-regulation (indicated with the prefix 'u') of reaction fluxes. For the synthetic models, we down-regulated $\mathrm{v}_{2}, \mathrm{v}_{3}$, and $\mathrm{v}_{4}$ by multiplying their constraint equation parameters $(a$ and $b$ parameters in all approaches and also including the $c$ parameter in the LK-DFBA (NLR) approach) by $0.5 \times$ (which restricts their maximum allowable flux value) and up-regulated these pathways by doubling the constraint equation parameters. The pathways and reactions to be perturbed in the L. lactis ${ }^{2,19-22}$ and E. coli $^{23-27}$ models were chosen based on previous literature. Reactions in the L. lactis model (lactate dehydrogenase, phosphofructokinase, acetate kinase, mannitol 1-phosphatase) were down-regulated to $0.1 \times$ their original parameter values (since completely knocking out reactions would often produce infeasible solutions for the linear program) and up-regulated to $2 \times$ their original parameter values, magnitudes that were necessary to effect significant perturbations to the system's behavior. Reactions in the E. coli model (pyruvate kinase, phosphoglucose isomerase, glyceraldehyde-3-phosphate dehydrogenase, phosphofructokinase, triose-phosphate isomerase, ribulosephosphate epimerase, phosphoglucomutase) were down-regulated to $0.1 \times$ and up-regulated to $2 \times$ their original parameter values.

Generating noisy data. Noise was introduced into the system using the original noiseless data (50 time points) and a down-sampling of that dataset into nT time points evenly spaced over the time interval of interest. Both metabolite and flux values were then replaced with a random value drawn from the normal distribution $N_{i, k} \sim\left(y_{i}\left(t_{k}\right), \operatorname{CoV} \cdot y_{i}\left(t_{k}\right)\right)$, where $y_{i}\left(t_{k}\right)$ is the value of species (metabolite or flux) $i$ at time point $k$, and CoV is a coefficient of variance. For each sampling frequency and $\mathrm{CoV}$ condition, ten noisy datasets were generated.

\section{Results}

Fitting and predicting phenotypes in synthetic models. We generated twenty random sets of parameters and initial conditions for the kinetic equations in the synthetic model to examine the utility of the new constraint approaches across models of the same topology but different parameter sets. We produced in silico metabolite concentration and flux data over a time interval of ten seconds by solving the ODEs in each synthetic system. The four constraint approaches were used for parameterization of LK-DFBA models to the twenty datasets. The fitted LK-DFBA models were then simulated over the same interval using the initial conditions for each respective synthetic system to compare against the original ODE data. This process was performed on both noiseless $(\mathrm{nT}=50, \mathrm{CoV}=0)$ and noise-added data with different sampling frequencies $(\mathrm{nT}=50$ or 15$)$ and levels of noise $(\mathrm{CoV}=0.05$ or 0.15$)$.

For the noiseless cases (Fig. 2A), the best-fitting constraint approach on the wild-type data (dark green boxes with X's) varied across the different models. All four approaches performed best for at least one of the models. Given that these models all have the same topology and just different parameter values, one might have expected they would be sufficiently similar that one single type of constraint would perform best across all or most models. The fact that different constraints fit different models better may be due to different qualitative behaviors being evident in the metabolic dynamics in the different models. Since all parameters were resampled in each model, they might access fundamentally different regimes of concentration or flux values and thus be better fit by different types of constraints. Similar trends were evident in noisy data (representative example in Fig. 2B), where the optimal constraint approach varied across the different models (boxes with X's in Fig. 2B).

Since a primary goal of building metabolic models is to predict the behavior of systems in conditions beyond those in which they are trained, we then tested the ability of each LK-DFBA model trained on wild-type data with different constraint approaches to predict the effects of defined genetic perturbations. We down- and up-regulated the $\mathrm{v}_{2}, \mathrm{v}_{3}$, and $\mathrm{v}_{4}$ pathways in the original kinetic equations by multiplying the kinetic coefficient parameters ( $a$ parameters in the inset of Fig. 1 ) by $0.5 \times$ or $2 \times$, respectively, and generating new ODE data to compare to LK-DFBA predictions. We then simulated the LK-DFBA model after adjusting the fitted LK-DFBA constraints to reflect the down- or up-regulation by multiplying the kinetics constraint parameters by $0.5 \times$ and $2 \times$, respectively. The normalized root mean square error (NRMSE; see Supplementary Methods) between the predicted LK-DFBA metabolite concentrations and the ODE concentration data from the perturbed synthetic models was then calculated.

Using noiseless training and test data, we observed that the best constraint approach for the wild-type training data (WT) was also the best for the test data across the majority of perturbations (dV2 through uV4), indicated by the clustering of dark green boxes in the rows for the constraint approach containing the box with an X for each model (Fig. 2A). The NRMSE heatmap with quantitative error values can be found in Fig. S1. This suggests that while perturbations that affect the entire network (i.e., by resampling all parameter values) can often lead to different constraint approaches being optimal, single perturbations yield sufficiently similar behavior to allow the same constraint approach to be optimal for both the training and the test data. 
A.

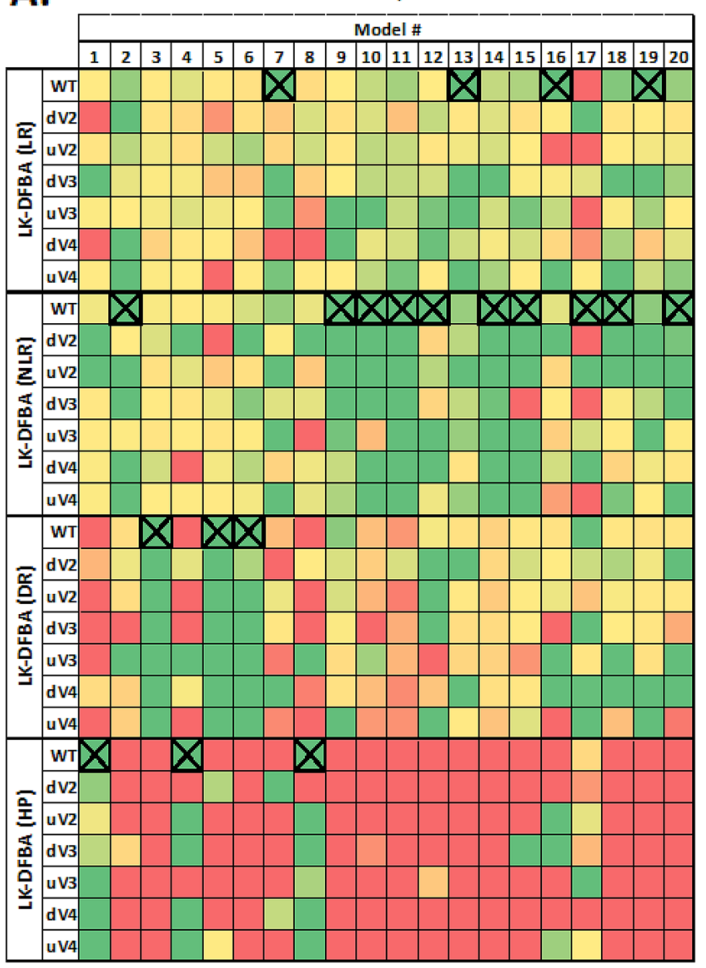

B.

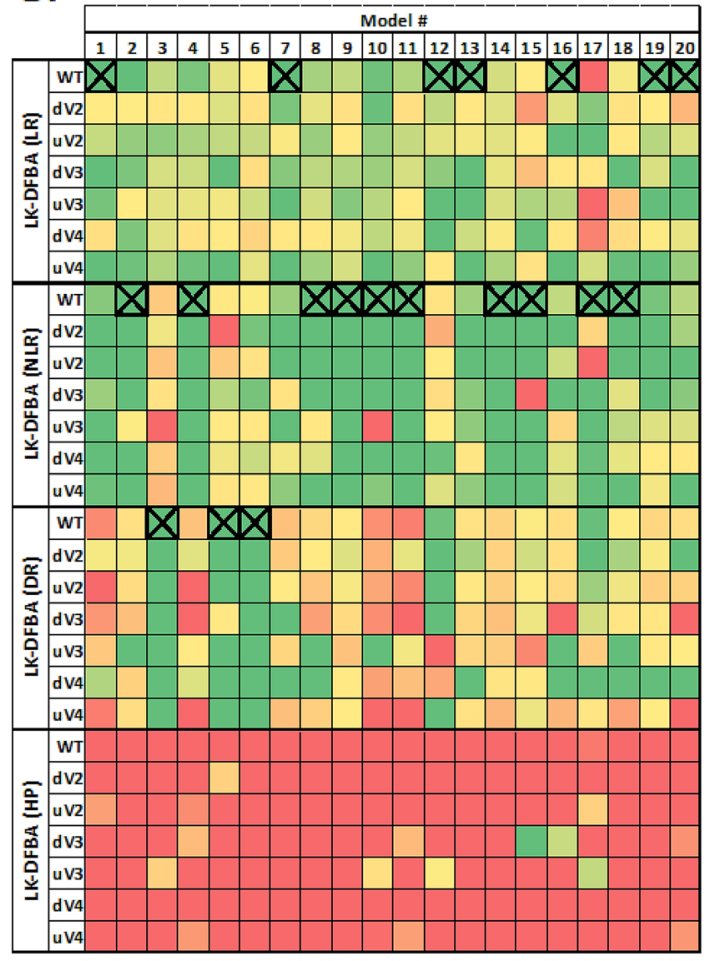

High NRMSE

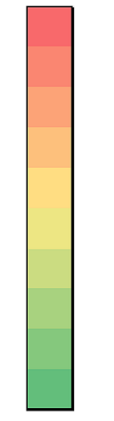

Low NRMSE

Figure 2. NRMSE heatmap of LK-DFBA approaches on different synthetic models. Each constraint approach was used to fit parameters to wild-type (WT) data and then used to simulate the WT system and in silico genetic perturbations with fluxes $\mathrm{v}_{2}, \mathrm{v}_{3}$, or $\mathrm{v}_{4}$ down- or up-regulated. The best constraint approach for each WT model is indicated with an X. Dark green boxes represent the lowest NRMSE within each genetic perturbation for each synthetic model, while dark red boxes represent the highest NRMSE (meaning that the dynamic range of the color scale varies for each perturbation for each synthetic model to better convey the relative performance of different methods). Panel A shows results for noiseless data with a sampling frequency of 50 time points. Panel B shows results for noisy datasets with a sampling frequency of 15 time points and with noise added at a $\mathrm{CoV}$ of 0.15 . The average NRMSE of 10 noisy datasets is shown in the heatmap. The same NRMSE heatmaps with explicitly annotated error values can be found in Figs. S1 and S5.

When using noisy data, similar trends were observed (representative example in Fig. 2B). While the best constraint approach for WT noisy data was not always the same as the best approach for noiseless data, the best constraint approach for a given noisy WT dataset was still the best in over half the cases for predicting the impacts of in silico genetic perturbations in the same model ( $\mathrm{dV} 2$ through uV4). Interestingly, noisy data negatively affected the performance of LK-DFBA (HP) to a much greater extent than the other approaches, which caused LK-DFBA (HP) to never be identified as the best approach under the most realistic conditions of $\mathrm{nT}=15$ and $\mathrm{CoV}=0.15$ (nor for almost any other noisy data condition, except for one (Fig. S2)). NRMSE heatmaps with quantitative error values for the different sampling frequencies and noise levels can be found in Figs. S2-S5.

We also tested the effect of smoothing the noisy ( $\mathrm{nT}=15, \mathrm{CoV}=0.15)$ metabolite concentration and flux time course profiles by fitting to a previously described ${ }^{28}$ impulse function (Fig. S6). Smoothing the noisy data often led to lower error of the final model but required increased computation time for estimating the parameters of the impulse function and in certain cases can actually increase error if a specific dataset deviates significantly from all of the profiles that an impulse function can capture. The best constraint approach for WT smoothed data was the same as for unsmoothed data in 19 of the 20 models. As with the unsmoothed cases, the best constraint approach for smoothed data was consistent between WT and the majority of in silico genetic perturbations, and there were no cases where LK-DFBA (HP) performed the best (and it was the worst out of the four approaches in 123 out of 140 cases) for smoothed data.

Fitting and predicting phenotypes in L. lactis and E. coli models. For the L. lactis model, we tested the four constraint approaches on noiseless data and noisy data under different conditions ( $\mathrm{nT}=50$ or 15 , $\mathrm{CoV}=0.05$ or 0.15 ). On the noiseless data, the best constraint approach for the WT system was LK-DFBA (HP), which also had the lowest NRMSE when predicting the results of perturbations to five different genes (Fig. 3A). At high sampling frequencies and low noise $(\mathrm{nT}=50, \mathrm{CoV}=0.05)$, LK-DFBA $(\mathrm{HP})$ still performed the best, but as more noise was added or lower sampling frequencies were used, LK-DFBA (NLR) overtook it to become the optimal approach. This is consistent with the findings described above for the small synthetic systems where LK-DFBA (HP) can produce low NRMSE with noiseless data but has difficulties under more realistic conditions. 
A. L. lactis

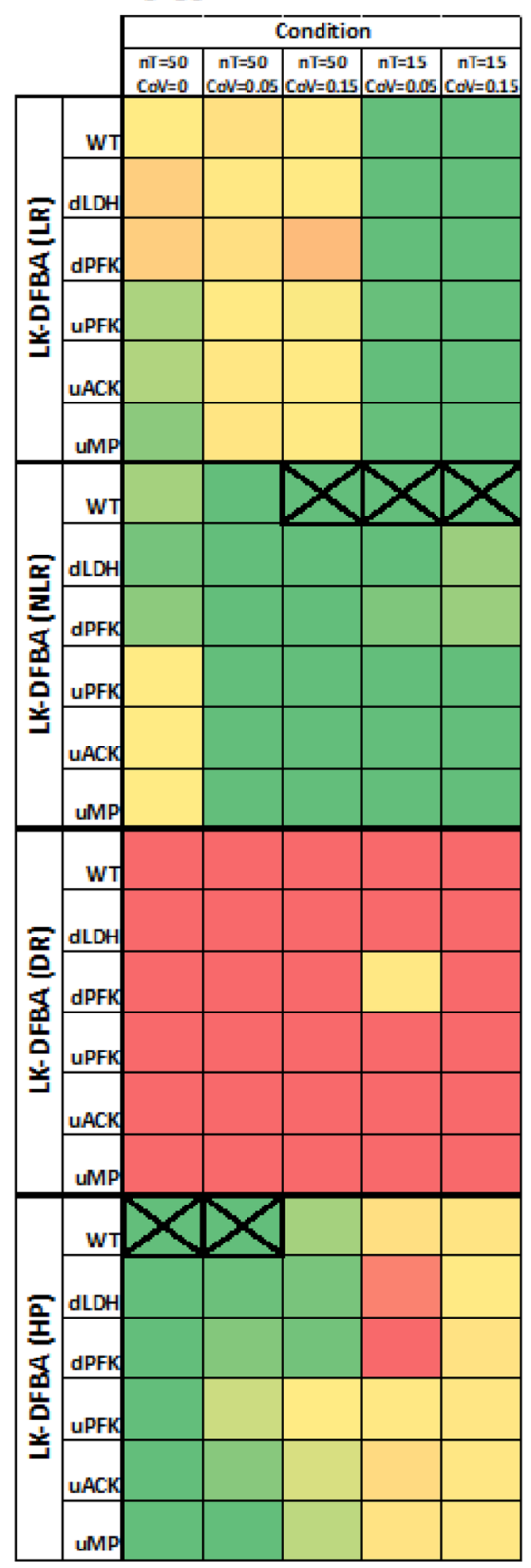

B. E. coli

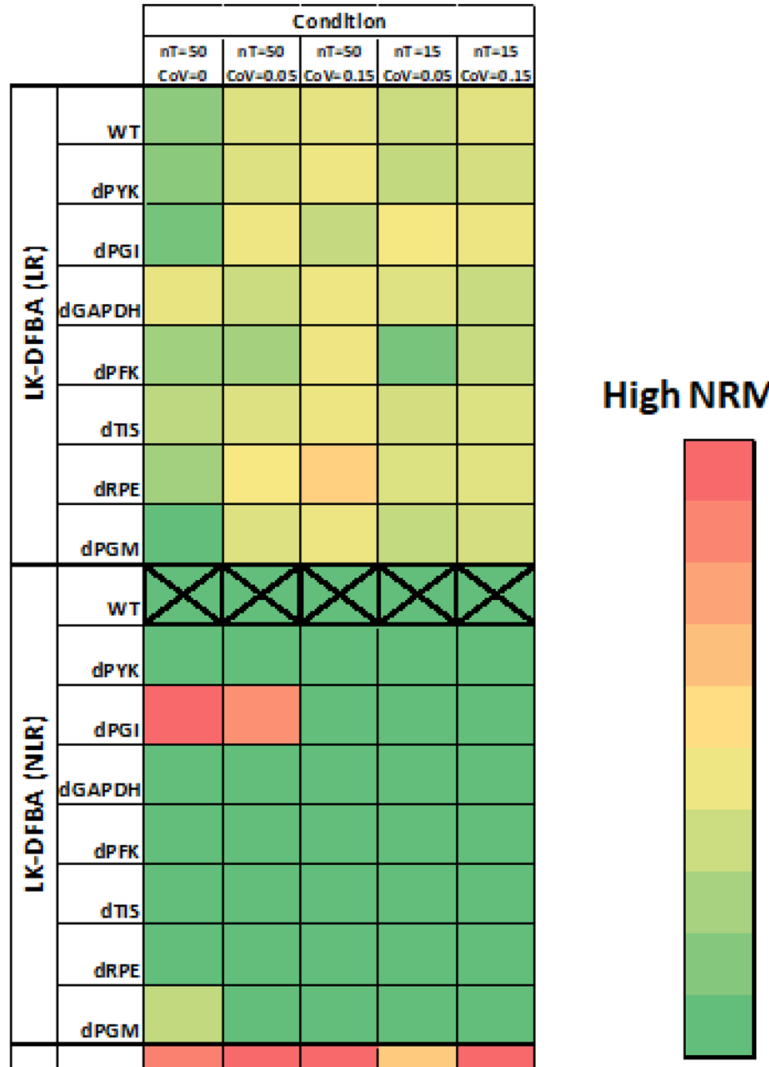

Low NRMSE

Figure 3. NRMSE heatmaps of constraint approaches on L. lactis and E. coli models. Each constraint approach was used to fit parameters to wild-type (WT) data and then used to simulate the WT system and the system with in silico genetic perturbations with literature-reported important pathways down- or up-regulated. The best constraint approach for each model is indicated with an X. Dark green boxes represent the lowest NRMSE within each phenotype for each model, while dark red boxes represent the highest NRMSE. Both the L. lactis (A) and E. coli (B) heatmaps show the mean of 10 noisy datasets, except for the noiseless condition (leftmost columns in each heatmap). The same NRMSE heatmaps with explicitly annotated error values can be found in Fig. S7. 
A.
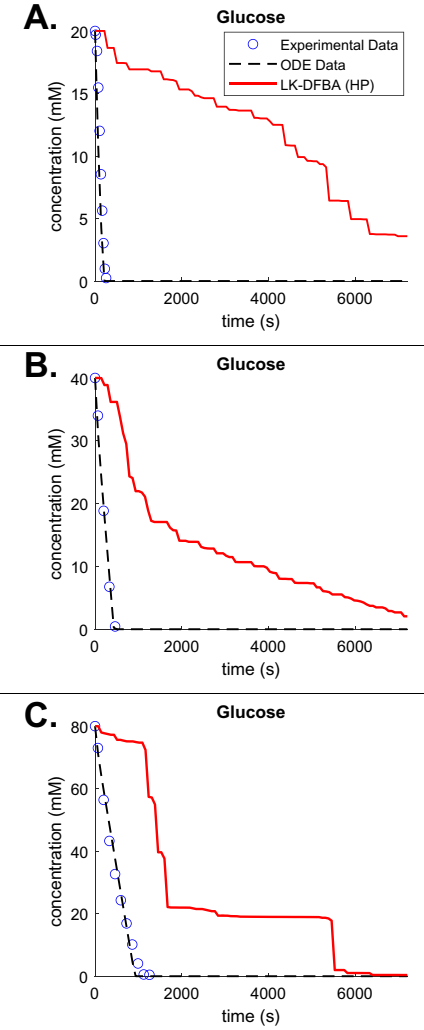
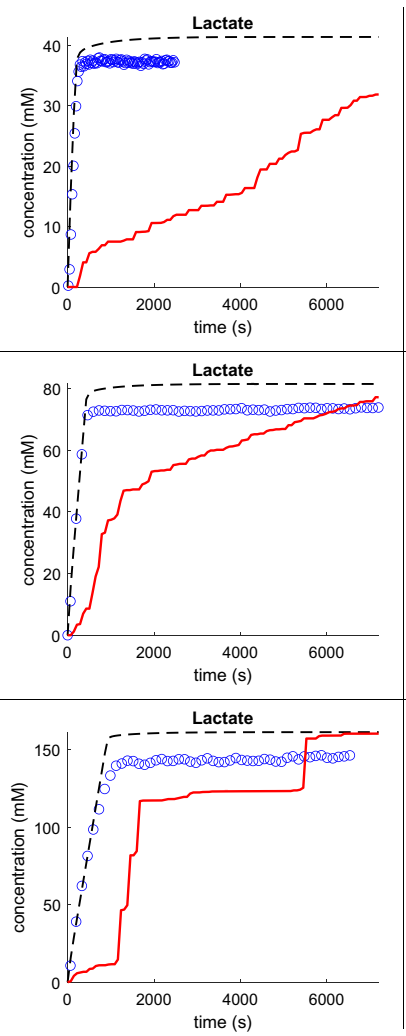
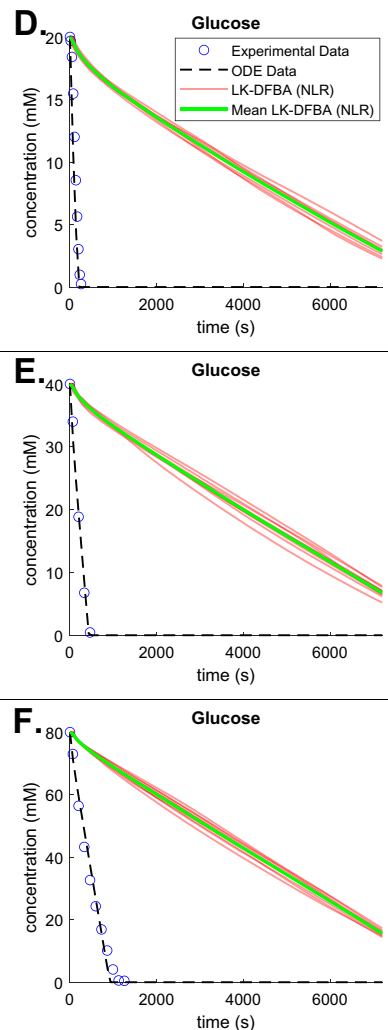
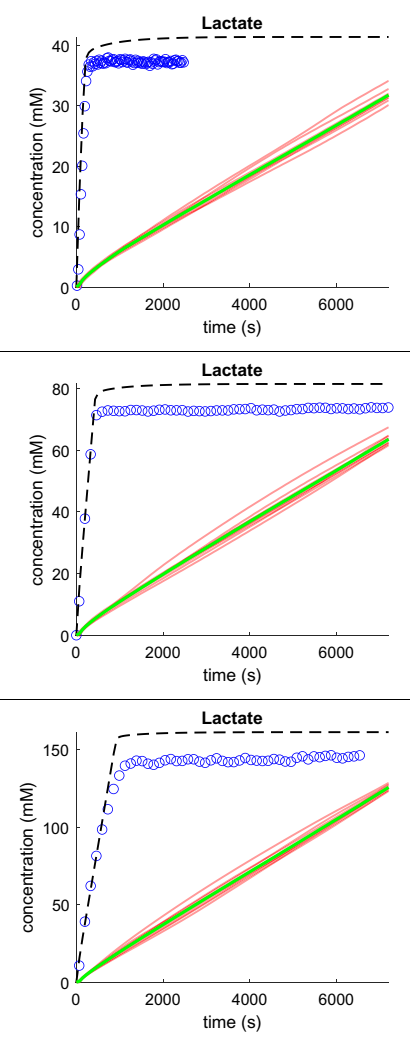

Figure 4. Comparison of LK-DFBA metabolite concentration predictions against ODE data and L. lactis experimental data when fitted to noiseless and noisy ODE data. Panels A, B, and C depict concentration profiles for LK-DFBA (HP) (solid red line) and the ODE model (dashed black line) compared to experimental data (blue circles) for initial glucose concentrations of $20 \mathrm{mM}, 40 \mathrm{mM}$, and $80 \mathrm{mM}$, respectively, when LK-DFBA is fitted to noiseless data. Panels D, E, and F depict concentration profiles for LK-DFBA (NLR) on 10 noisy datasets $(\mathrm{nT}=15, \mathrm{CoV}=0.15)$ and the ODE model compared to experimental data. The mean concentration profile (solid green line) is shown with each of the concentration profiles (solid red lines) from the 10 noisy datasets.

As with the L. lactis model, we tested all constraint approaches on both noiseless and noisy data from the $E$. coli model under different conditions ( $\mathrm{nT}=50$ or $15, \mathrm{CoV}=0.05$ or 0.15 ). For this model, LK-DFBA (NLR) was the best constraint approach for noiseless data (Fig. 3B). Noisy E. coli data produced the same results: for all noisy conditions, LK-DFBA (NLR) was optimal for the WT system. It was also optimal for 27 of the 28 in silico genetic perturbations, showing once again that the same constraint approach that was optimal for the WT system at a given sampling condition tends to be optimal for the perturbed systems, supporting the generalizability of selecting a constraint approach based only on WT data.

We also perturbed the original parameters and initial conditions (drawing from the random normal distribution $N_{i} \sim\left(p_{i}, p_{i}\right)$ and taking the absolute value, where $p_{i}$ is the original value of the $i$ th parameter) of the $E$. coli model to create five new models with the same topology (more information about parameter randomization can be found in the Supplementary Methods). As with the twenty different versions of the small synthetic system, we found that across these models with identical topology and only different parameter values, the best constraint approach varied substantially (Fig. S8). Again, the rates of individual reactions and how they affect overall model dynamics appear to be critical factors in determining the optimal constraint approach.

Improved LK-DFBA predictions yield qualitative consistency with experimental L. lactis metabolite concentration data. To further assess how well LK-DFBA performs when predicting different phenotypes, we compared the predictions of LK-DFBA to available experimental data-the first time such a comparison has been performed for this modeling framework. The previously described ODE-based $L$. lactis model was originally parameterized using experimental metabolite time course data from L. lactis cultures grown with an initial glucose concentration of $40 \mathrm{mM}^{2}$ and validated by comparison to some experimental data from cultures grown at initial concentrations of $20 \mathrm{mM}$ and $80 \mathrm{mM}$ glucose. Here, we similarly fitted all LKDFBA approaches to data generated by the ODE model at $40 \mathrm{mM}$ glucose and then, using the best constraint approach (based on the NRMSE compared to the ODE data), simulated the LK-DFBA model at $20 \mathrm{mM}$ and $80 \mathrm{mM}$ initial concentrations of glucose for validation.

Figure 4A-C depict the metabolite concentrations predicted by LK-DFBA (HP) (the best approach for noiseless data in the L. lactis model) when trained on noiseless data. For multiple initial glucose concentrations, LK-DFBA (HP) captured the general qualitative trends of glucose (depletion) and lactate (accumulation), two 
key metabolites in L. lactis that are often studied ${ }^{29,30}$. For cofactor metabolites that participate in many different reactions, such as ATP, NAD $(\mathrm{H})$, and inorganic phosphate (Fig. S9), it was more challenging for LK-DFBA (HP) to predict their concentration profiles over the simulation interval, which is a problem found in other modeling frameworks ${ }^{7}$. Although LK-DFBA's predictions were overall not as smooth or quantitatively accurate as the ODE model, this is to be expected due to the lack of a validated objective function for this constraint-based model; the objective function we used was a gross approximation that likely does not reflect the cell's true "goal", and it is known that the objective function can significantly affect the predictions of FBA approaches. Nevertheless, as presented here, LK-DFBA can still qualitatively track important metabolite dynamics even when using a crude objective function. This is important to note, as many organisms that are not well-studied have no readily available objective function to use.

Figure 4D-F depict the glucose and lactate concentration profiles predicted by LK-DFBA (NLR) (the best approach for noisy data in the L. lactis model) after being fitted to 10 noisy datasets generated by the ODE model and simulated at $20 \mathrm{mM}, 40 \mathrm{mM}$, and $80 \mathrm{mM}$ initial glucose, respectively. Again, the LK-DFBA framework captured qualitative trends of major metabolites such as glucose and lactate, though unsurprisingly not as accurately as when noiseless data are used and with difficulties predicting cofactor concentrations (Fig. S10). Because LK-DFBA (NLR) contains quadratic constraints, its results are smoother compared to the other LKDFBA approaches, which helped it predict some metabolites, such as PEP, arguably better than in the noiseless case. Furthermore, LK-DFBA (NLR) is less susceptible to noise for some metabolites, such as glucose and lactate, as observed in predicting similar time courses across the 10 noisy datasets. This could be advantageous if one is modeling a system with multiple noisy data sets and requires consistent predictions for certain metabolites. Likewise, if only using a single dataset, LK-DFBA (NLR) can ensure that these metabolic profiles would not dramatically change if a different dataset had been used. Other methods, such as the original LK-DFBA (LR) approach, can result in more varied predictions (Fig. S11) depending on the noisy dataset used; some appear to produce better predictions than LK-DFBA (NLR), while others are worse (though all predictions follow the same trends). These observations reiterate that the best approach is dependent on the systems and datasets being studied, so having multiple LK-DFBA approaches available is an improvement over only using the LK-DFBA (LR) framework.

Changes in LK-DFBA flux profiles due to gene knockouts are correlated with experimental $E$. coli steady-state flux data. We also compared the predictions of the best LK-DFBA approach (based on the NRMSE compared to the ODE data) on the E. coli model to experimental steady-state flux data obtained through gene knockout experiments by Ishii et al. ${ }^{24}$. Because the Chassagnole model, which LK-DFBA is fitted to, only encompasses central carbon metabolism, we focused on 13 gene knockouts (plus the wildtype system) and 14 fluxes that are included in both the Chassagnole model and the Ishii steady-state flux results. We used the dilution rate of $0.2 \mathrm{~h}^{-1}$ for all experimental data. To emulate a gene knockout in the LK-DFBA model, we down-regulated the pathway(s) that correspond with the gene by multiplying the parameters of the relevant constraints by $0.1 \times$ instead of completely removing the reaction, as we found that this sufficiently reduced the possible flux reaction rate, while completely knocking out a reaction (i.e. setting the relevant constraints to 0 ) would sometimes cause infeasible problems. Additionally, it is not uncommon for enzymatic activity to remain in a biological pathway after single gene knockouts due to paralogous enzymes and enzyme promiscuity. Because the LK-DFBA predictions do not reach steady-state for the simulation time examined in this work and our previous work (ten seconds), we instead used the average flux of the predicted time course to describe how LK-DFBA's predictions change from the wild-type to gene knockout phenotype (see "Methods"). We also examined longer simulation times that allowed LK-DFBA and the Chassagnole model to reach steady-state, but found that the kinetic model was not optimized for long simulation times (Fig. S12). Nevertheless, the average flux before and after a gene knockout should reflect whether the reaction rate generally increases or decreases across the studied time interval after a system perturbation. We used a Pearson correlation to determine if the average flux profiles of 14 reactions predicted by LK-DFBA and the original kinetic model behaved similarly to the experimental data before and after a gene knockout (see Supplementary Methods for details). Both the LK-DFBA and kinetic models were not fit to the Ishii data, which allowed us to compare the models to an independent set of experimental E. coli data. A similar method has been used previously by Lima et al. to compare multiple E. coli models, including the Chassagnole model, to the Ishii dataset ${ }^{31}$.

To evaluate how our framework compares to E. coli experimental data, we examined LK-DFBA (NLR), as it was the best approach in the case of low sampling frequency and high noise (Fig. 3B). Figure 5 shows the average Pearson correlation of the LK-DFBA (NLR) flux predictions (after being fitted to ten noisy datasets with $\mathrm{nT}=15$ and $\mathrm{CoV}=0.15)$ and the average correlation of the ODE model flux predictions with the experimental steady-state flux data. When comparing LK-DFBA (NLR) predictions to the experimental data, we calculated correlation values greater than 0.55 in all but two cases and correlations greater than 0.7 in 8 out of 14 cases. These correlations were very similar to the correlations yielded by the ODE-based model. In 11 out of 14 phenotypes, the correlations calculated for LK-DFBA outperformed or were within $5 \%$ of the correlations calculated with the ODE-based model. LK-DFBA was comparatively even better when the simulation interval was extended to $600 \mathrm{~s}$ (Fig. S12). These results support the potential promise of LK-DFBA approaches for predictivity comparable to that of standard models but with the additional benefits (including relative model simplicity and potential scalability) that accrue from using a LP-based formulation. 


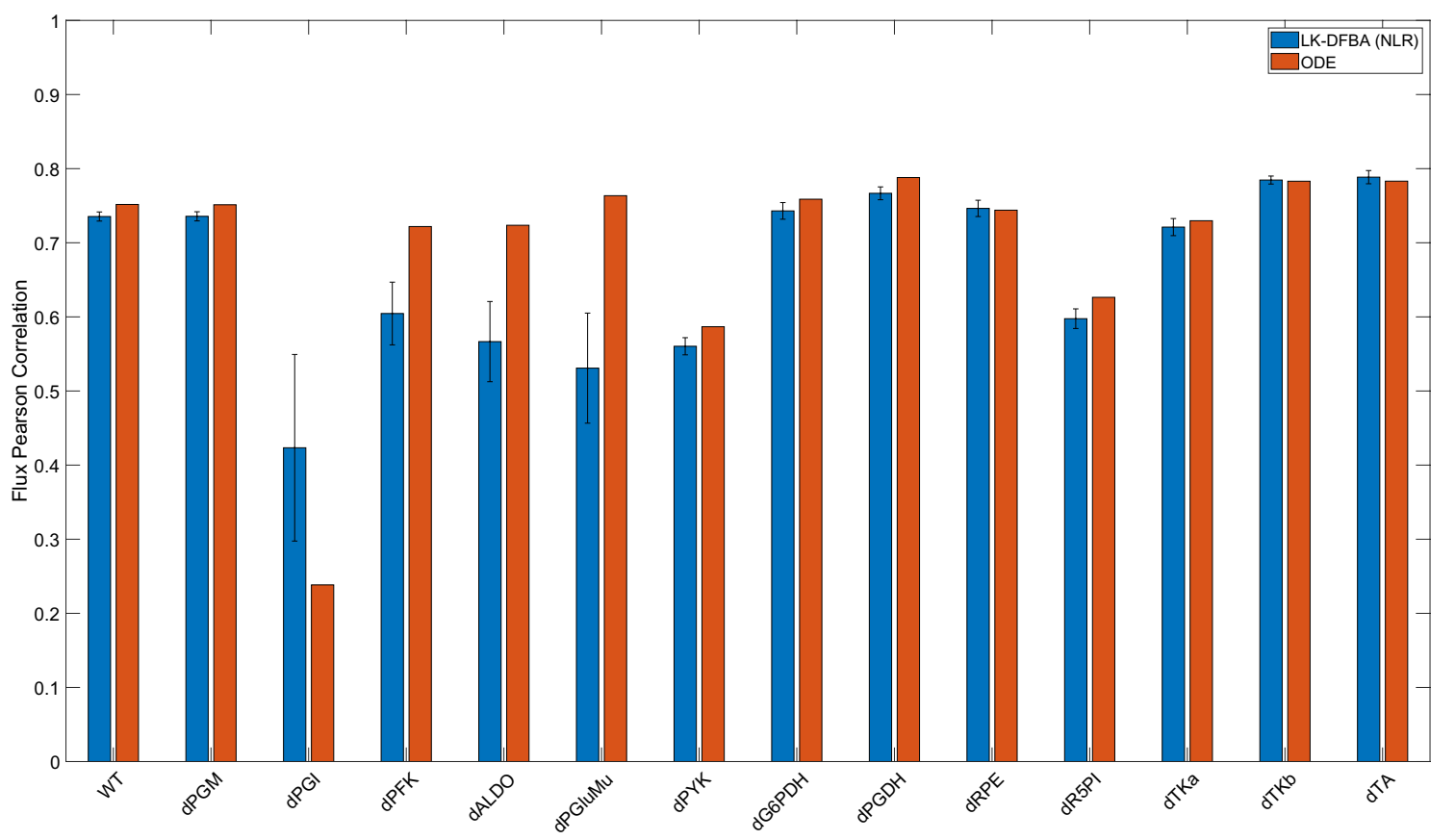

Figure 5. Pearson correlation coefficients of LK-DFBA and ODE model flux predictions with $E$. coli experimental data. LK-DFBA (NLR) was the best approach when fitting on low sampling frequency $(\mathrm{nT}=15)$ and high noise $(\mathrm{CoV}=0.15)$ data. Blue and red bars represent LK-DFBA (NLR) and ODE model mean correlations, respectively, between the average predicted flux profiles and experimental steady-state flux data for the wildtype system and various gene knockout conditions. Gene knockouts in the LK-DFBA and ODE-based models were simulated by down-regulating relevant pathways. Error bars for LK-DFBA represent one standard deviation ( $\mathrm{N}=10$ runs).

\section{Discussion}

At the outset of performing this work, we intended to identify an LK-DFBA constraint approach to improve upon the originally published framework that used only linear kinetics constraints. Instead, we have demonstrated that the best constraint approach depends on both the topology and the parameters of the system being modeled. Despite each of the 20 small synthetic models having the exact same stoichiometric and regulatory topology, the optimal LK-DFBA approach varied across these 20 models, and for the majority of the models one of the new constraint approaches performed the best. This finding suggests that the emergent dynamics from the collective metabolic reactions are just as important as the topology of the system in determining which constraint approach best fits data from the system. It also supports the potential benefit of having multiple types of constraints to choose from, as done in this work, to enable more accurate modeling of any given system.

These findings from synthetic systems were reinforced by analysis of biological systems based on E. coli and L. lactis metabolism. While LK-DFBA (HP) performed the best on L. lactis noiseless data, LK-DFBA (NLR) performed the best on E. coli noiseless data. (We do note, though, that LK-DFBA (NLR) was superior for both systems under the lowest sampling frequency and highest noise data conditions). We further confirmed that topology is not the sole factor that determines the optimal constraint approach for a given system by randomizing parameters in the E. coli model (Fig. S8): again, the best constraint approach varied across these topologically identical new models. This finding has particular relevance to the development of metabolic models for organisms beyond those few that are widely studied: many metabolic pathways are topologically well-conserved across many species (e.g. central carbon metabolism ${ }^{1}$ ), though the kinetic and regulatory parameters in these pathways can be vastly different. Thus, having multiple constraint approaches to choose from will improve the ability to model different systems using LK-DFBA.

Critically, the potential use of multiple types of constraints for a given model does not appear to contribute substantially to overfitting. While the best constraint approach varied across different model parameterizations and topologies, the best approach for a given model for predicting metabolic phenotypes due to up- or down-regulating different pathways was consistent across the majority of perturbations. This trend remained true whether using noiseless data, data with low sampling frequency and high noise, or noisy data that had been smoothed. These results instill confidence that the best constraint approach found when fitting to a wild-type metabolic system will also be the best approach when predicting changes to that system, meaning that an approach entailing the selection of the best-fitting of multiple constraint frameworks is viable, likely to be successful, and unlikely to contribute substantially to overfitting. We speculate that the reason for the success of this approach may be due to the robustness of the emergent metabolic dynamics of a system. Resampling all of the kinetic parameters in a system (as we did for 20 synthetic models and 5 E. coli models) can yield qualitatively different metabolite or flux profiles exhibiting qualitatively different behaviors that are better captured by different types of constraints. When 


\section{Dynamic Flux Estimation}

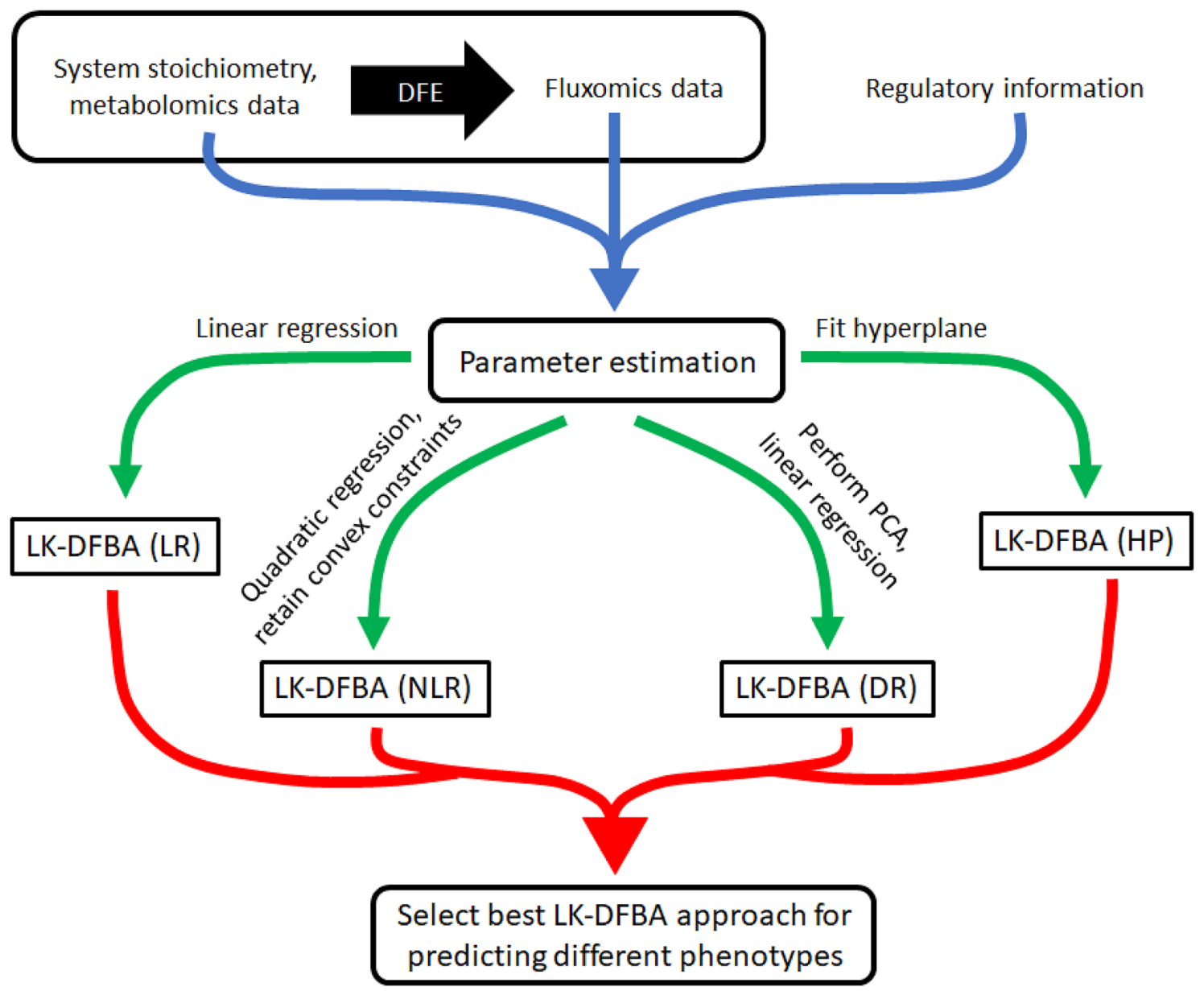

Figure 6. Workflow for selecting the best constraint approach for LK-DFBA when modeling metabolic systems. Dynamic Flux Estimation (DFE) is applied to the system stoichiometry and available metabolomics data to infer instantaneous fluxes. The system stoichiometry, metabolomics data, inferred flux data, and system regulatory information are then used to estimate parameters for each LK-DFBA approach (blue arrow). Using multiple constraint approaches (green arrows), four different LK-DFBA models are created and tested for their respective abilities to recapitulate training data. The model with the lowest error is selected and can be used for future in silico predictions (red arrow).

individual genes or pathways are down- or up-regulated, though, it is common for only the nearest neighboring metabolites or pathways to be significantly affected, meaning that the emergent behavior from the system would not change too greatly and thus the same constraint approach would be optimal. To easily construct the optimal LK-DFBA model for a given biological system, we envision the workflow presented in Fig. 6. After compiling the relevant system stoichiometry, regulatory structure, and metabolomics and fluxomics data, one can fit each of the four LK-DFBA approaches to the data and determine which constraint approach is optimal. Again, based on our findings, this optimal constraint approach fitted to the wild-type dataset is the most likely to work the best for predicting the results of model perturbations.

While the best constraint approach was dependent on the system being modeled, we did find some possible trends among the four constraint methods. In several of the synthetic models and the L. lactis system, LK-DFBA (HP) was observed to be the best constraint approach when using noiseless data. However, as lower sampling frequencies were used and more noise was added to the data, LK-DFBA (HP) decreased in performance and was never the best approach under the most difficult conditions in any of the systems assessed, which indicates that LK-DFBA (HP) may be most useful when data are readily available and close to their true values. We also found that the NRMSEs of LK-DFBA (LR) were often similar to the NRMSEs of LK-DFBA (NLR), which is to be expected because the two approaches share similar constraints except LK-DFBA (NLR) includes an additional quadratic term when a concave quadratic constraint is feasible for a particular interaction. It is interesting that LK-DFBA (NLR) was not always better than LK-DFBA (LR), which suggests that the quadratic constraints could sometimes be allowing overfitting of the data. We also note that the NRMSEs for LK-DFBA (DR) were not consistently similar to the results for any of the other constraint methods, likely due to the fundamental differences between the data reduction approach and the basic constraint approaches. 
Beyond these investigations into the utility and robustness of new types of constraints for LK-DFBA, we also compared LK-DFBA predictions to experimental data. Using ODE models and experimental data from $L$. lactis and E. coli, we found that LK-DFBA can effectively predict qualitative trends in concentration profiles of some important metabolites. While we have previously shown that LK-DFBA captures metabolite dynamics in synthetic data generated by ODE models, this is the first time LK-DFBA predictions have been validated with experimental data. For key metabolites that are important inputs and outputs of the system (e.g. carbon sources or end products), LK-DFBA can qualitatively predict if their concentration profiles are expected to decrease or increase, which is an important capability when assessing how perturbations affect a system. Cofactors, on the other hand, are more difficult to model using LK-DFBA but are still predicted to be within an order of magnitude of the experimental data in most cases. This capability could be useful when assessing levels of accumulating toxic metabolites or cofactor imbalances if exact concentrations are not necessary. While these results hold some promise, LK-DFBA does not achieve the same quantitative accuracy as a kinetic model. Because LK-DFBA does not use mechanistic equations that describe the underpinnings of each kinetic reaction, LK-DFBA is not able to closely track metabolite concentrations when compared to ODE-based models. However, the novel kinetics constraints in a constraint-based framework have the potential to enable LK-DFBA to capture metabolic trends more efficiently, which with further work could enable better scale-up to modeling complex systems.

We also found that LK-DFBA flux profile predictions were similarly correlated with experimental flux data from genetic knockout experiments compared to the ODE-based model. We note, though, that this comparable predictivity is limited by the fact that LK-DFBA was trained using ODE-generated data; if it had instead been fitted to actual metabolomics and fluxomics time course data used in the Ishii experiments (which is not available), these correlation values could possibly be even higher. Similarly, an improved objective function over the reasonable but arbitrary and unoptimized one used here could also lead to significant improvements in the performance of LK-DFBA.

By showing for the first time that LK-DFBA can predict changes in metabolite concentrations and flux profiles qualitatively, these results support LK-DFBA's potential for eventual use as a metabolic modeling tool once more of its limitations have been addressed. While many ODE-based modeling approaches require specific kinetic equations for each flux reaction, LK-DFBA is more generalized. With four types of kinetics constraints that account for different biological interaction phenomena between metabolites and fluxes, we have made LK-DFBA more amenable to different systems. Additionally, applying the four LK-DFBA approaches to these models of L. lactis and E. coli has established that our framework can handle various biological systems without the need for computationally taxing parameter estimation steps (using only regression for parameter estimation, as in this work). Because each of the four LK-DFBA approaches maintains an easily solvable LP or QCP structure, LK-DFBA is a prime candidate for scaling up to model a variety of genome-scale systems while also capturing their metabolite dynamics.

While the addition of new constraint approaches has significantly improved the original LK-DFBA (LR) framework, there are still several next steps that will need to be explored and areas where LK-DFBA must be improved before it can become a widely-used modeling framework. In this work, we have shown that LK-DFBA can model small synthetic and biological systems, but we have not yet validated our work on a larger system. LK-DFBA requires metabolomics and fluxomics data (if DFE is not possible) for each metabolite and reaction in the system, which is difficult to obtain experimentally. We previously determined that LK-DFBA models are slightly affected when data for a single metabolite is missing ${ }^{11}$, but if a significant number of metabolite time course profiles are unavailable, this could more severely impact the framework. While simulating these data could be a viable option to characterize such behaviors, there are so few genome-scale kinetic models that even this alternative remains challenging. We initially attempted to use the genome-scale kinetic model described by Khodayari and Maranas ${ }^{7}$, but the model uses normalized concentrations while LK-DFBA requires absolute concentrations. In the future, a large-scale synthetic system with hundreds of metabolites and reactions could be used to further test our framework.

In addition to testing a larger system, the effect of introducing multiple perturbations (either as gene overexpressions, knockdowns, or a combination of both) should be examined. While we have shown that a single perturbation usually does not change the best LK-DFBA constraint approach compared to the best approach on the wild-type system, we have also determined that different sets of kinetic parameters greatly affect the results of each constraint approach. Perturbing multiple reactions in a system will likely have a greater effect on LKDFBA performance compared to a single perturbation. Examining different strengths of up- or down-regulated reactions (i.e. multiplying constraint parameters by values other than $0.1 \times$ or $2 \times$ ) can also be explored further to determine how predicted concentrations and fluxes are affected.

To improve the parameters in each of the new constraint approaches, a secondary optimization step can be used, as in the LK-DFBA (LR +) approach. However, this step can be computationally expensive, and as the system gets bigger the computational burden of parameter estimation in a large nonlinear system may become intractable. The most immediately scalable approach would be LK-DFBA (LR), and thus for this work we focused on developing new constraint approaches that could still be useful without parameter optimization. However, similar analyses in the context of LK-DFBA $(\mathrm{LR}+)$ could also be instructive.

In addition, as previously noted the objective function used in LK-DFBA is also a ripe target for future efforts to improve this modeling framework. Here we have chosen objective functions that lead to the maximization of putatively important fluxes, but unlike many other constraint-based models, there was no specific biomass or other objective flux to use. For the L. lactis system, we assessed an objective function that maximized all efflux reactions (similar to the E. coli system), but found that it led to worse performance (Fig. S13). Despite the worse performance, we still observed that the best performing constraint approach (which was once again LK-DFBA (HP) for noiseless data even with the different objective function) was still consistent between the wildtype and perturbed systems. Optimizing the weight of each flux or metabolite in the objective function could lead to even 
lower observed errors compared to experimental data and may also provide insight into the selective pressures on real biological systems.

\section{Conclusion}

In this work, we have shown that the LK-DFBA modeling framework can be improved by implementing more complex constraints with increased biological relevance. We showed that there is no single best LK-DFBA constraint approach for all models, and that the optimal approach depends not just on the topology of the biochemical system but also its kinetics and parameters. Importantly, though, the constraint approach that performs the best in recapitulating training data outperforms other constraint approaches at predicting the results of metabolic perturbations on the same system, suggesting that selecting between multiple different types of constraints improves model predictivity with limited contributions to overfitting. With these new constraint approaches, we are able to model a variety of metabolic systems more accurately than if we were to just use the original LK-DFBA (LR) method. Moreover, based on comparisons to experimental data, we showed that the improved LK-DFBA approaches can reasonably capture the qualitative dynamics of important metabolites and fluxes of interest to researchers. While these predictions may not be smooth or quantitative, the qualitative prediction of trends of metabolite dynamics in response to major perturbations gives valuable insight for designing experiments that implement these genetic modifications. Moreover, we expect this computational framework to (with future effort) provide the potential for computationally reasonable scale-up to the genome scale. While the acquisition of quality metabolomics and fluxomics data to build the constraints in LK-DFBA is still a challenge, the work we have presented here lays the groundwork needed to take full advantage of these types of datasets as they become increasingly more readily available.

\section{Data availability}

The code and datasets generated during the current study are available at https://github.com/gtstylab/lk-dfbaconstraints.

Received: 23 June 2021; Accepted: 8 December 2021

Published online: 14 January 2022

\section{References}

1. Chassagnole, C., Noisommit-Rizzi, N., Schmid, J. W., Mauch, K. \& Reuss, M. Dynamic modeling of the central carbon metabolism of Escherichia coli. Biotechnol. Bioeng. 79, 53-73 (2002).

2. Costa, R. S., Hartmann, A., Gaspar, P., Neves, A. R. \& Vinga, S. An extended dynamic model of Lactococcus lactis metabolism for mannitol and 2,3-butanediol production. Mol. Biosyst. 10, 628-639 (2014).

3. Gottstein, W., Olivier, B. G., Bruggeman, F. J. \& Teusink, B. Constraint-based stoichiometric modelling from single organisms to microbial communities. J. R. Soc. Interface. 13, 20160627 (2016).

4. Lularevic, M., Racher, A. J., Jaques, C. \& Kiparissides, A. Improving the accuracy of flux balance analysis through the implementation of carbon availability constraints for intracellular reactions. Biotechnol. Bioeng. 116, 2339-2352 (2019).

5. Hynne, F., Dano, S. \& Sorensen, P. G. Full-scale model of glycolysis in Saccharomyces cerevisiae. Biophys. Chem. 94, 121-163 (2001).

6. Nolan, R. P. \& Lee, K. Dynamic model of CHO cell metabolism. Metab. Eng. 13, 108-124 (2011).

7. Khodayari, A. \& Maranas, C. D. A genome-scale Escherichia coli kinetic metabolic model k-ecoli457 satisfying flux data for multiple mutant strains. Nat. Commun. 7, 13806 (2016).

8. Smallbone, K., Simeonidis, E., Swainston, N. \& Mendes, P. Towards a genome-scale kinetic model of cellular metabolism. BMC Syst. Biol. 4, 6 (2010).

9. Srinivasan, S., Cluett, W. R. \& Mahadevan, R. Constructing kinetic models of metabolism at genome-scales: A review. Biotechnol. J. 10, 1345-1359 (2015).

10. Stanford, N. J. et al. Systematic construction of kinetic models from genome-scale metabolic networks. PLoS ONE 8, e79195 (2013).

11. Dromms, R. A., Lee, J. Y. \& Styczynski, M. P. LK-DFBA: A linear programming-based modeling strategy for capturing dynamics and metabolite-dependent regulation in metabolism. BMC Bioinform. 21, 93 (2020).

12. Burgard, A. P., Pharkya, P. \& Maranas, C. D. Optknock: A bilevel programming framework for identifying gene knockout strategies for microbial strain optimization. Biotechnol. Bioeng. 84, 647-657 (2003).

13. Chen, W. W., Niepel, M. \& Sorger, P. K. Classic and contemporary approaches to modeling biochemical reactions. Genes Dev. 24, 1861-1875 (2010).

14. Voit, E. O. Biochemical systems theory: A review. ISRN Biomath. 2013, 1-53 (2013).

15. Goel, G., Chou, I. C. \& Voit, E. O. System estimation from metabolic time-series data. Bioinformatics 24, 2505-2511 (2008).

16. Maranas, C. D. \& Zomorrodi, A. R. Optimization Methods in Metabolic Networks (Wiley, 2016).

17. Wold, S., Esbensen, K. \& Geladi, P. Principal component analysis. Chemometr. Intell. Lab. 2, 37-52 (1987).

18. Costa, R. S., Verissimo, A. \& Vinga, S. KiMoSys: A web-based repository of experimental data for KInetic MOdels of biological SYStems. BMC Syst. Biol. 8, 85 (2014).

19. Gaspar, P., Neves, A. R., Gasson, M. J., Shearman, C. A. \& Santos, H. High yields of 2,3-butanediol and Mannitol in Lactococcus lactis through engineering of $\mathrm{NAD}(+)$ cofactor recycling. Appl. Environ. Microb. 77, 6826-6835 (2011).

20. Neves, A. R., Ramos, A., Shearman, C., Gasson, M. J. \& Santos, H. Catabolism of mannitol in Lactococcus lactis MG1363 and a mutant defective in lactate dehydrogenase. Microbiology (Reading) 148, 3467-3476 (2002).

21. Oh, E. et al. Dynamic modeling of lactic acid fermentation metabolism with Lactococcus lactis. J. Microbiol. Biotechnol. 21, $162-169$ (2011).

22. Wisselink, H. W. et al. Overproduction of heterologous mannitol 1-phosphatase: A key factor for engineering mannitol production by Lactococcus lactis. Appl. Environ. Microbiol. 71, 1507-1514 (2005).

23. Asmawaty, T. et al. Modeling and simulation of the main metabolism in Escherichia coli and its several single-gene knockout mutants with experimental verification. Microbiol. Cell Fact. 9, 1-21 (2010).

24. Ishii, N. et al. Multiple high-throughput analyses monitor the response of E. coli to perturbations. Science 316, 593-597 (2007).

25. Kurata, H. \& Sugimoto, Y. Improved kinetic model of Escherichia coli central carbon metabolism in batch and continuous cultures. J. Biosci. Bioeng. 125, 251-257 (2018).

26. Long, C. P., Gonzalez, J. E., Sandoval, N. R. \& Antoniewicz, M. R. Characterization of physiological responses to 22 gene knockouts in Escherichia coli central carbon metabolism. Metab. Eng. 37, 102-113 (2016). 
27. Usui, Y. et al. Investigating the effects of perturbations to pgi and eno gene expression on central carbon metabolism in Escherichia coli using 13C metabolic flux analysis. Microbiol. Cell Fact. 11, 1-16 (2012).

28. Dromms, R. A. \& Styczynski, M. P. Improved metabolite profile smoothing for flux estimation. Mol. Biosyst. 11, 2394-2405 (2015).

29. Kleerebezem, M. et al. Metabolic engineering of Lactococcus lactis: the impact of genomics and metabolic modelling. J. Biotechnol. 98, 199-213 (2002).

30. Papagianni, M. Metabolic engineering of lactic acid bacteria for the production of industrially important compounds. Comput. Struct. Biotechnol. J. 3, e201210003 (2012).

31. Lima, A. P., Baixinho, V., Machado, D. \& Rocha, I. A comparative analysis of dynamic models of the central carbon metabolism of Escherichia coli. IFAC Papers Online 49, 270-276 (2016).

\section{Author contributions}

J.Y.L. conceived of the study, participated in the design of the study, carried out computational experiments, analyzed experimental results, and helped to draft the manuscript. M.P.S. conceived of the study, participated in the design of the study, analyzed experimental results, and helped to draft the manuscript. All authors read and approved of the final manuscript.

\section{Competing interests}

The authors declare no competing interests.

Additional information

Supplementary Information The online version contains supplementary material available at https://doi.org/ 10.1038/s41598-021-03934-0.

Correspondence and requests for materials should be addressed to M.P.S.

Reprints and permissions information is available at www.nature.com/reprints.

Publisher's note Springer Nature remains neutral with regard to jurisdictional claims in published maps and institutional affiliations.

(c) (i) Open Access This article is licensed under a Creative Commons Attribution 4.0 International License, which permits use, sharing, adaptation, distribution and reproduction in any medium or format, as long as you give appropriate credit to the original author(s) and the source, provide a link to the Creative Commons licence, and indicate if changes were made. The images or other third party material in this article are included in the article's Creative Commons licence, unless indicated otherwise in a credit line to the material. If material is not included in the article's Creative Commons licence and your intended use is not permitted by statutory regulation or exceeds the permitted use, you will need to obtain permission directly from the copyright holder. To view a copy of this licence, visit http://creativecommons.org/licenses/by/4.0/.

(C) The Author(s) 2022 